\title{
Peptoniphilus indolicus
}

National Cancer Institute

\section{Source}

National Cancer Institute. Peptoniphilus indolicus. NCI Thesaurus. Code C86904.

A species of anaerobic, Gram-positive, cocci shaped bacteria assigned to the phylum Firmicutes. This species is nonmotile, non-spore forming, indole positive, catalase and urease negative, reduces nitrate, produces butyric acid, gas from peptone-yeast-glucose medium and propionate from lactate, coagulase and indole, and does not ferment carbohydrates or liquefy gelatin. P. indolicus is found in cattle, swine, and rats and is pathogenic to humans and possibly animals. 